\title{
Instantons: topological aspects
}

\author{
Marcos Jardim \\ IMECC - UNICAMP \\ Departamento de Matemática \\ Caixa Postal 6065 \\ 13083-970 Campinas-SP, Brazil
}

October 31, 2018

\begin{abstract}
Keywords: Gauge Theory; differential geometry of vector bundles with connections; instantons; anti-self-dual connections; moduli spaces; YangMills equation; Yang-Mills functional; ADHM construction; Nahm's equations; monopoles; Hitchin's equations.

Cross-references: Moduli spaces (62); Instantons in gauge theory (72); Mathematical uses of gauge theory (75); Index theorems (149); Donaldson invariants (255); Characteristic classes (354); Differential geometry (386); Finite dimensional algebras and quivers (418).
\end{abstract}

\section{Introduction}

Let $X$ be a closed (connected, compact without boundary) smooth manifold of dimension 4 , provided with a Riemannian metric denoted by $g$. Let $\Omega_{X}^{p}$ denote space of smooth $p$-forms on $X$, i.e. the sections of $\wedge^{p} T X$. The Hodge operator acting on $p$-forms:

$$
*: \Omega_{X}^{p} \rightarrow \Omega_{X}^{4-p}
$$

satisfies $*^{2}=(-1)^{p}$. In particular, $*$ splits $\Omega_{X}^{2}$ into two sub-spaces $\Omega_{X}^{2, \pm}$ with eigenvalues \pm 1 :

$$
\Omega_{X}^{2}=\Omega_{X}^{2,+} \oplus \Omega_{X}^{2,-}
$$

Note also that this decomposition is an orthogonal one, with respect to the inner product:

$$
\left\langle\omega_{1}, \omega_{2}\right\rangle=\int_{X} \omega_{1} \wedge * \omega_{2} .
$$

A 2-form $\omega$ is said to be self-dual if $* \omega=\omega$ and it is said to be anti-self-dual if $* \omega=-\omega$. Any 2 -form $\omega$ can be written as the sum

$$
\omega=\omega^{+}+\omega^{-}
$$


of its self-dual $\omega^{+}$and anti-self-dual $\omega^{-}$components.

Now let $E$ be a complex vector bundle over $X$ as above, provided with a connection $\nabla$, regarded as a $\mathbb{C}$-linear operator

$$
\nabla: \Gamma(E) \rightarrow \Gamma(E) \otimes \Omega_{X}^{1}
$$

satisfying the Leibnitz rule:

$$
\nabla(f \sigma)=f \nabla \sigma+\sigma \otimes d f
$$

for all $f \in C^{\infty}(X)$ and $\sigma \in \Gamma(E)$. Its curvature $F_{\nabla}=\nabla \circ \nabla$ is a 2-form with values in $\operatorname{End}(E)$, i.e. $F_{\nabla} \in \Gamma(\operatorname{End}(E)) \otimes \Omega_{X}^{2}$, satisfying the Bianchi identity $\nabla F_{\nabla}=0$.

The Yang-Mills equation is:

$$
\nabla * F_{\nabla}=0
$$

It is a $2^{\text {nd }}$-order non-linear equation on the connection $\nabla$. It amounts to a non-abelian generalization of Maxwell equations, to which it reduces when $E$ is a line bundle; the four components of $\nabla$ are interpreted as the electric and magnetic potentials.

An instanton on $E$ is a smooth connection $\nabla$ whose curvature $F_{\nabla}$ is anti-selfdual as a 2-form, i.e. it satisfies:

$$
F_{\nabla}^{+}=0 \text { i.e. } * F_{\nabla}=-F_{\nabla} .
$$

The instanton equation is still non-linear (it is linear only if $E$ is a line bundle), but it is only $1^{\text {st }}$-order on the connection.

Note that if $F_{\nabla}$ is either self-dual or anti-self-dual as a 2-form, then the YangMills equation is automatically satisfied:

$$
* F_{\nabla}= \pm F_{\nabla} \Rightarrow \nabla * F_{\nabla}= \pm \nabla F_{\nabla}=0
$$

by the Bianchi identity. In other words, instantons are particular solutions of the Yang-Mills equation. Furthermore, while the Yang-Mills equation (2) makes sense over any Riemannian manifold, the instanton equation (3) is well-defined only in dimension 4.

A gauge transformation is a bundle automorphism $g: E \rightarrow E$ covering the identity. The set of all gauge transformations of a given bundle $E \rightarrow X$ form a group through composition, called the gauge group and denoted by $\mathcal{G}(E)$. The gauge group acts on the set of all smooth connections on $E$ by conjugation:

$$
g \cdot \nabla=g^{-1} \nabla g .
$$

It is then easy to see that (3) is gauge invariant condition, since $F_{g \cdot \nabla}=g^{-1} F_{\nabla} g$. The anti-self-duality equation (3) is also conformally invariant: a conformal change in the metric does not change the decomposition (1), so it preserves self-dual and anti-self-dual 2-forms. 
The topological charge $k$ of the instanton $\nabla$ is defined by the integral:

$$
\begin{aligned}
k & =-\frac{1}{8 \pi^{2}} \int_{X} \operatorname{tr}\left(F_{\nabla} \wedge F_{\nabla}\right) \\
& =c_{2}(E)-\frac{1}{2} c_{1}(E)^{2}
\end{aligned}
$$

where the second equality follows from Chern-Weil theory.

If $X$ is a smooth, non-compact, complete Riemannian manifold, an instanton on $X$ is an anti-self-dual connection for which the integral (4) converges. Note that in this case, $k$ as above need not be an integer; however it is expected to always be quantized, i.e. always a multiple of some fixed (rational) number which depends only on the base manifold $X$.

Summary. This note is organized as follows. After revisiting the variational approach to the anti-self-duality equation (3), we study instantons over the simplest possible Riemannian 4-manifold, $\mathbb{R}^{4}$ with the flat Euclidean metric. We present 't Hooft's explicit solutions (Section 3), the ADHM construction (Section (4) and its dimensional reductions to $\mathbb{R}^{3}, \mathbb{R}^{2}$ and $\mathbb{R}$ (Section 5 ). We conclude in Section 6 by explaining the construction of the central object of study in gauge theory, the instanton moduli spaces.

\section{Variational aspects of Yang-Mills equation.}

Given a fixed smooth vector bundle $E \rightarrow X$, let $\mathcal{A}(E)$ be the set of all (smooth) connections on $E$. The Yang-Mills functional is defined by

$$
\begin{gathered}
\mathrm{YM}: \mathcal{A}(E) \rightarrow \mathbb{R} \\
\mathrm{YM}(\nabla)=\left\|F_{\nabla}\right\|_{L^{2}}^{2}=\int_{M} \operatorname{tr}\left(F_{\nabla} \wedge * F_{\nabla}\right)
\end{gathered}
$$

The Euler-Lagrange equation for this functional is exactly the Yang-Mills equation (2). In particular, self-dual and anti-self-dual connections yield critical points of the Yang-Mills functional.

Splitting the curvature into its self-dual and anti-self-dual parts, we have

$$
\mathrm{YM}(\nabla)=\left\|F_{\nabla}^{+}\right\|_{L^{2}}^{2}+\left\|F_{\nabla}^{-}\right\|_{L^{2}}^{2}
$$

It is then easy to see that every anti-self-dual connection $\nabla$ is an absolute minimum for the Yang-Mills functional, and that $\mathrm{YM}(\nabla)$ coincides with the topological charge (4) of the instanton $\nabla$ times $8 \pi^{2}$.

One can construct, for various 4-manifolds but most interestingly for $X=S^{4}$, solutions of the Yang-Mills equations which are neither self-dual nor anti-selfdual. Such solutions do not minimize (5). Indeed, at least for gauge group $S U(2)$ or $S U(3)$, it can be shown that there are no other local minima: any critical point which is neither self-dual nor anti-self-dual is unstable and must be a "saddle point" 3 . 


\section{Instantons on Euclidean space}

Let $X=\mathbb{R}^{4}$ with the flat Euclidean metric, and consider a hermitian vector bundle $E \rightarrow \mathbb{R}^{4}$. Any connection $\nabla$ on $E$ is of the form $d+A$, where $d$ denotes the usual de Rham operator and $A \in \Gamma(\operatorname{End}(E)) \otimes \Omega_{\mathbb{R}^{4}}^{1}$ is a 1 -form with values in the endomorphisms of $E$; this can be written as follows:

$$
A=\sum_{k=1}^{4} A_{k} d x^{k} \quad, \quad A_{k}: \mathbb{R}^{4} \rightarrow \mathfrak{u}(r)
$$

In the Euclidean coordinates $x_{1}, x_{2}, x_{3}, x_{4}$, the anti-self-duality equation (3) is given by:

$$
F_{12}=F_{34} \quad, \quad F_{13}=-F_{24} \quad, \quad F_{14}=F_{23}
$$

where

$$
F_{i j}=\frac{\partial A_{j}}{\partial x_{i}}-\frac{\partial A_{i}}{\partial x_{j}}+\left[A_{i}, A_{j}\right] .
$$

The simplest explicit solution is the charge $1 S U(2)$-instanton on $\mathbb{R}^{4}$. The connection 1-form is given by:

$$
A_{0}=\frac{1}{1+|x|^{2}} \cdot \operatorname{Im}(q d \bar{q})
$$

where $q$ is the quaternion $q=x_{1}+x_{2} \mathbf{i}+x_{3} \mathbf{j}+x_{4} \mathbf{k}$, while Im denotes the imaginary part of the product quaternion; we are regarding $\mathbf{i}, \mathbf{j}, \mathbf{k}$ as a basis of the Lie algebra $\mathfrak{s u}(2)$; from this, one can compute the curvature:

$$
F_{A_{0}}=\left(\frac{1}{1+|x|^{2}}\right)^{2} \cdot \operatorname{Im}(d q \wedge d \bar{q})
$$

We see that the action density function

$$
\left|F_{A_{0}}\right|^{2}=\left(\frac{1}{1+|x|^{2}}\right)^{2}
$$

has a bell-shaped profile centered at the origin and decaying like $r^{-4}$.

Let $t_{\lambda, y}: \mathbb{R}^{4} \rightarrow \mathbb{R}^{4}$ be the isometry given by the composition of a translation by $y \in \mathbb{R}^{4}$ with a homothety by $\lambda \in \mathbb{R}^{+}$. The pullback connection $t_{\lambda, y}^{*} A_{0}$ is still anti-self-dual; more explicitly:

$$
\begin{gathered}
A_{\lambda, y}=t_{\lambda, y}^{*} A_{0}=\frac{\lambda^{2}}{\lambda^{2}+|x-y|^{2}} \cdot \operatorname{Im}(q d \bar{q}) \\
\text { and } F_{A_{\lambda, y}}=\left(\frac{\lambda^{2}}{\lambda^{2}+|x-y|^{2}}\right)^{2} \cdot \operatorname{Im}(d q \wedge d \bar{q}) .
\end{gathered}
$$

Note that the action density function $\left|F_{A}\right|^{2}$ has again a bell-shaped profile centered at $y$ and decaying like $r^{-4}$; the parameter $\lambda$ measures the concentration of 
the energy density function, and can be interpreted as the "size" of the instanton $A_{\lambda, y}$.

Instantons of topological charge $k$ can be obtained by "superimposing" $k$ basic instantons, via the so-called 't Hooft ansatz. Consider the function $\rho: \mathbb{R}^{4} \rightarrow \mathbb{R}$ given by:

$$
\rho(x)=1+\sum_{j=1}^{k} \frac{\lambda_{j}^{2}}{\left(x-y_{j}\right)^{2}},
$$

where $\lambda_{j} \in \mathbb{R}$ and $y_{j} \in \mathbb{R}^{4}$. Then the connection 1-form $A=A_{\mu} d x_{\mu}$ with coefficients

$$
A_{\mu}=i \sum_{\nu=1}^{4} \bar{\sigma}_{\mu \nu} \frac{\partial}{\partial x_{\nu}} \ln (\rho(x))
$$

is anti-self dual; here, $\bar{\sigma}_{\mu \nu}$ are the matrices given by $(\mu, \nu=1,2,3)$ :

$$
\bar{\sigma}_{\mu \nu}=\frac{1}{4 i}\left[\sigma_{\mu}, \sigma_{\nu}\right] \bar{\sigma}_{\mu 4}=\frac{1}{2} \sigma_{\mu}
$$

where $\sigma_{\mu}$ are the Pauli matrices.

The connection (8) correspond to $k$ instantons centered at points $y_{i}$ with size $\lambda_{i}$. The basic instanton (6) is exactly (modulo gauge transformation) what one obtains from (8) for the case $k=1$. The 't Hooft instantons form a $5 k$ parameter family of anti-self-dual connections.

$S U(2)$-instantons are also the building blocks for instantons with general structure group [2]. Let $G$ be a compact semi-simple Lie group, with Lie algebra $\mathfrak{g}$. Let $\phi: \mathfrak{s u}(2) \rightarrow \mathfrak{g}$ be any injective Lie algebra homomorphism. If $A$ is an anti-self-dual $S U(2)$-connection 1 -form, then it is easy to see that $\phi(A)$ is an anti-self-dual $G$-connection 1 -form. Using (8) as an example, we have that:

$$
A=i \sum_{\mu, \nu} \phi\left(\bar{\sigma}_{\mu \nu}\right) \frac{\partial}{\partial x_{\nu}} \ln (\rho(x)) d x_{\mu}
$$

is a $G$-instanton on $\mathbb{R}^{4}$.

While this guarantees the existence of $G$-instantons on $\mathbb{R}^{4}$, note that the instanton (9) might be reducible (e.g. $\phi$ can simply be the obvious inclusion of $\mathfrak{s u}(2)$ into $\mathfrak{s u}(n)$ for any $n)$ and that its charge depends on the choice of representation $\phi$. Furthermore, it is not clear whether every $G$-instanton can be obtained in this way, as the inclusion of a $S U(2)$-instanton through some representation $\phi: \mathfrak{s u}(2) \rightarrow \mathfrak{g}$.

\section{The ADHM construction}

All $S U(r)$-instantons on $\mathbb{R}^{4}$ can be obtained through a remarkable construction due to Atiyah, Drinfeld, Hitchin and Manin. It starts by considering hermitian vector spaces $V$ and $W$ of dimension $c$ and $r$, respectively, and the following data:

$$
B_{1}, B_{2} \in \operatorname{End}(V), i \in \operatorname{Hom}(W, V), \quad j \in \operatorname{Hom}(V, W)
$$


so-called ADHM data. Assume moreover that $\left(B_{1}, B_{2}, i, j\right)$ satisfy the $A D H M$ equations:

$$
\begin{aligned}
{\left[B_{1}, B_{2}\right]+i j } & =0 \\
{\left[B_{1}, B_{1}^{\dagger}\right]+\left[B_{2}, B_{2}^{\dagger}\right]+i i^{\dagger}-j^{\dagger} j } & =0
\end{aligned}
$$

Now consider the following maps

$$
\begin{aligned}
& \alpha: V \times \mathbb{R}^{4} \rightarrow(V \oplus V \oplus W) \times \mathbb{R}^{4} \\
& \beta:(V \oplus V \oplus W) \times \mathbb{R}^{4} \rightarrow V \times \mathbb{R}^{4}
\end{aligned}
$$

given as follows ( $\mathbf{1}$ denotes the appropriate identity matrix):

$$
\begin{gathered}
\alpha\left(z_{1}, z_{2}\right)=\left(\begin{array}{c}
B_{1}+z_{1} \mathbf{1} \\
B_{2}+z_{2} \mathbf{1} \\
j
\end{array}\right) \\
\beta\left(z_{1}, z_{2}\right)=\left(\begin{array}{ccc}
-B_{2}-z_{2} \mathbf{1} & B_{1}+z_{1} \mathbf{1} & i
\end{array}\right)
\end{gathered}
$$

where $z_{1}=x_{1}+i x_{2}$ and $z_{2}=x_{3}+i x_{4}$ are complex coordinates on $\mathbb{R}^{4}$. The maps (12) and (13) should be understood as a family of linear maps parameterized by points in $\mathbb{R}^{4}$.

A straightforward calculation shows that the ADHM equation (10) imply that $\beta \alpha=0$ for every $\left(z_{1}, z_{2}\right) \in \mathbb{R}^{4}$. Therefore the quotient $E=\operatorname{ker} \beta / \operatorname{im} \alpha=\operatorname{ker} \beta \cap$ ker $\alpha^{\dagger}$ forms a complex vector bundle over $\mathbb{R}^{4}$ or rank $r$ whenever $\left(B_{1}, B_{2}, i, j\right)$ is such that $\alpha$ is injective and $\beta$ is surjective for every $\left(z_{1}, z_{2}\right) \in \mathbb{R}^{4}$.

To define a connection on $E$, note that $E$ can be regarded as a sub-bundle of the trivial bundle $(V \oplus V \oplus W) \times \mathbb{R}^{4}$. So let $\iota: E \rightarrow(V \oplus V \oplus W) \times \mathbb{R}^{4}$ be the inclusion, and let $P:(V \oplus V \oplus W) \times \mathbb{R}^{4} \rightarrow E$ be the orthogonal projection onto $E$. We can then define a connection $\nabla$ on $E$ through the projection formula

$$
\nabla s=P \underline{d} \iota(s)
$$

where $\underline{d}$ denotes the trivial connection on the trivial bundle $(V \oplus V \oplus W) \times \mathbb{R}^{4}$. To see that this connection is anti-self-dual, note that projection $P$ can be written as follows

$$
P=1-\mathcal{D}^{\dagger} \Xi^{-1} \mathcal{D}
$$

where

$$
\begin{gathered}
\mathcal{D}:(V \oplus V \oplus W) \times \mathbb{R}^{4} \rightarrow(V \oplus V) \times \mathbb{R}^{4} \\
\mathcal{D}=\left(\begin{array}{c}
\beta \\
\alpha^{\dagger}
\end{array}\right)
\end{gathered}
$$

and $\Xi=\mathcal{D D}^{\dagger}$. Note that $\mathcal{D}$ is surjective, so that $\Xi$ is indeed invertible. Moreover, it also follows from (11) that $\beta \beta^{\dagger}=\alpha^{\dagger} \alpha$, so that $\Xi^{-1}=\left(\beta \beta^{\dagger}\right)^{-1} \mathbf{1}$. 
The curvature $F_{\nabla}$ is given by:

$$
\begin{aligned}
F_{\nabla} & =P\left(\underline{d}\left(\mathbf{1}-\mathcal{D}_{\mathrm{I}}^{\dagger} \Xi^{-1} \mathcal{D}\right) \underline{d}\right)=P\left(\underline{d} \mathcal{D}^{\dagger} \Xi^{-1}(\underline{d} \mathcal{D})\right)= \\
& =P\left(\left(\underline{d} \mathcal{D}^{\dagger}\right) \Xi^{-1}(\underline{d} \mathcal{D})+\mathcal{D}^{\dagger} \underline{d}\left(\Xi^{-1}(\underline{d} \mathcal{D})\right)=\left(\underline{d} \mathcal{D}^{\dagger}\right) \Xi^{-1}(\underline{d} \mathcal{D})\right.
\end{aligned}
$$

for $P\left(\mathcal{D}^{\dagger} \underline{d}\left(\Xi^{-1}(\underline{d} \mathcal{D})\right)\right)=0$ on $E=\operatorname{ker} \mathcal{D}$. Since $\Xi^{-1}$ is diagonal, we conclude that $F_{\nabla}$ is proportional to $d \mathcal{D}^{\dagger} \wedge d \mathcal{D}$, as a 2 -form.

It is then a straightforward calculation to show that each entry of $d \mathcal{D}^{\dagger} \wedge d \mathcal{D}$ belongs to $\Omega^{2,-}$.

The extraordinary accomplishment of Atiyah, Drinfeld, Hitchin and Manin was to show that every instanton, up to gauge equivalence, can be obtained in this way; see e.g. [4. For instance, the basic $S U(2)$-instanton (6) is associated with the following data $(c=1, r=2)$ :

$$
B_{1}, B_{2}=0, i=\left(\begin{array}{l}
1 \\
0
\end{array}\right), j=\left(\begin{array}{ll}
0 & 1
\end{array}\right)
$$

Remark. The ADHM data $\left(B_{1}, B_{2}, i, j\right)$ is said to be stable if $\beta$ is surjective for every $\left(z_{1}, z_{2}\right) \in \mathbb{R}^{4}$, and it is said to be costable if $\alpha$ is injective for every $\left(z_{1}, z_{2}\right) \in \mathbb{R}^{4} . \quad\left(B_{1}, B_{2}, i, j\right)$ is regular if it is both stable and costable. The quotient:

$$
\text { \{regular solutions of (10) and (11) }\} / U(V)
$$

coincides with the moduli space of instantons of rank $r=\operatorname{dim} W$ and charge $c=\operatorname{dim} V$ on $\mathbb{R}^{4}$ (see below). It is also an example of a quiver variety (see the article "Finite dimensional algebras and quivers" by Alistair Savage), associated to the quiver consisting of two vertices $V$ and $W$, two loop-edges on the vertex $V$ and two edges linking $V$ to $W$, one in each direction.

\section{Dimensional reductions of the anti-self-dual Yang-Mills equation.}

As pointed out above, a connection on an hermitian vector bundle $E \rightarrow \mathbb{R}^{4}$ of rank $r$ can be regarded as 1 -form

$$
A=\sum_{k=1}^{4} A_{k}\left(x_{1}, \cdots, x_{4}\right) d x^{k} \quad, \quad A_{k}: \mathbb{R}^{4} \rightarrow \mathfrak{u}(r) .
$$

Assuming that the connection components $A_{k}$ are invariant under translation in one direction, say $x_{4}$, we can think of

$$
\underline{A}=\sum_{k=1}^{3} A_{k}\left(x_{1}, x_{2}, x_{3}\right) d x^{k}
$$

as a connection on a hermitian vector bundle over $\mathbb{R}^{3}$, with the fourth component $\phi=A_{4}$ being regarded as a bundle endomorphism $\phi: E \rightarrow E$, called a Higgs 
field. In this way, the anti-self-duality equation (3) reduces to the so-called Bogomolny (or monopole) equation:

$$
F_{\underline{A}}=* d \phi
$$

where $*$ is the Euclidean Hodge star in dimension 3 .

Now assume that the connection components $A_{k}$ are invariant under translation in two directions, say $x_{3}$ and $x_{4}$. Consider

$$
\underline{A}=\sum_{k=1}^{2} A_{k}\left(x_{1}, x_{2}\right) d x^{k}
$$

as a connection on a hermitian vector bundle over $\mathbb{R}^{2}$, with the third and fourth components combined into a complex bundle endomorphism:

$$
\Phi=\left(A_{3}+i \cdot A_{4}\right)\left(d x_{1}-i \cdot d x_{2}\right)
$$

taking values on 1-forms. The anti-self-duality equation (3) is then reduced to the so-called Hitchin's equations:

$$
\left\{\begin{array}{l}
F_{\underline{A}}=\left[\Phi, \Phi^{*}\right] \\
\bar{\partial}_{\underline{A}} \Phi=0
\end{array}\right.
$$

Conformal invariance of the anti-self-duality equation means that Hitchin's equations are well-defined over any Riemann surface.

Finally, assume that the connection components $A_{k}$ are invariant under translation in three directions, say $x_{2}, x_{3}$ and $x_{4}$. After gauging away the first component $A_{1}$, the anti-self-duality equations (3) reduce to the so-called Nahm's equations:

$$
\frac{d T_{k}}{d x_{1}}+\frac{1}{2} \sum_{j, l} \epsilon_{k j l}\left[T_{j}, T_{l}\right]=0, \quad j, k, l=\{2,3,4\}
$$

where each $T_{k}$ is regarded as a map $\mathbb{R} \rightarrow \mathfrak{u}(r)$.

Those interested in monopoles and Nahm's equations are referred to the survey [9] and the references therein. The best source for Hitchin's equations still are Hitchin's original papers [6]. A beautiful duality know as Nahm transform relates the various reductions of the anti-self-duality equation to periodic instantons; see the survey article [7].

It is also worth mentioning the book by Mason \& Woodhouse [8, where other interesting dimensional reductions of the anti-self-duality equation are discussed, providing a deep relation between instantons and the general theory of integrable systems.

\section{The instanton moduli space}

Now fix a rank $r$ complex vector bundle $E$ over a 4-dimensional Riemannian manifold $X$. Observe that the difference between any two connections is a linear 
operator:

$$
\left(\nabla-\nabla^{\prime}\right)(f \sigma)=f \nabla \sigma+\sigma \cdot d f-f \nabla^{\prime} \sigma-\sigma \cdot d f=f\left(\nabla-\nabla^{\prime}\right) \sigma .
$$

In other words, any two connections on $E$ differ by an endomorphism valued 1-form. Therefore, the set of all smooth connections on $E$, denoted by $\mathcal{A}(E)$, has the structure of an affine space over $\Gamma(\operatorname{End}(E)) \otimes \Omega_{M}^{1}$.

The gauge group $\mathcal{G}(E)$ acts on $\mathcal{A}(E)$ via conjugation:

$$
g \cdot \nabla:=g^{-1} \nabla g
$$

We can form the quotient set $\mathcal{B}(E)=\mathcal{A}(E) / \mathcal{G}(E)$, which is the set of gauge equivalence classes of connections on $E$.

The set of gauge equivalence classes of anti-self-dual connections on $E$ is a subset of $\mathcal{B}(E)$, and it is called the moduli space of instantons on $E \rightarrow X$. The subset of $\mathcal{M}_{X}(E)$ consisting of irreducible anti-self-dual connections is denoted $\mathcal{M}_{X}^{*}(E)$. Since the choice of a particular vector bundle within its topological class is immaterial, these sets are usually labeled by the topological invariants (Chern or Pontrjagyn classes) of the bundle $E$. For instance, $\mathcal{M}(r, k)$ denotes the moduli space of instantons on a rank $r$ complex vector bundle $E \rightarrow X$ with $c_{1}(E)=0$ and $c_{2}(E)=k>0$.

It turns out that $\mathcal{M}_{X}(E)$ can be given the structure of a Hausdorff topological space. In general, $\mathcal{M}_{X}(E)$ will be singular as a differentiable manifold, but $\mathcal{M}_{X}^{*}(E)$ can always be given the structure of a smooth Riemannian manifold.

We start by explaining the notion of a $L_{p}^{2}$ vector bundle. Recall that $L_{p}^{2}\left(\mathbb{R}^{n}\right)$ denotes the completion of the space of smooth functions $f: \mathbb{R}^{n} \rightarrow \mathbb{C}$ with respect to the norm:

$$
\|f\|_{L_{p}^{2}}^{2}=\int_{X}\left(|f|^{2}+|d f|^{2}+\cdots\left|d^{(p)} f\right|^{2}\right) .
$$

In dimension $n=4$ and for $p>2$, by virtue of the Sobolev embedding theorem, $L_{p}^{2}$ consists of continuous functions, i.e. $L_{p}^{2}\left(\mathbb{R}^{n}\right) \subset C^{0}\left(\mathbb{R}^{n}\right)$. So we define the notion of a $L_{p}^{2}$ vector bundle as a topological vector bundle whose transition functions are in $L_{p}^{2}$, where $p>2$.

Now fixed a $L_{p}^{2}$ vector bundle $E$ over $X$, we can consider the metric space $\mathcal{A}_{p}(E)$ of all connections on $E$ which can be represented locally on an open subset $U \subset X$ as a $L_{p}^{2}(U) 1$-form. In this topology, the subset of irreducible connections $\mathcal{A}_{p}^{*}(E)$ becomes an open dense subset of $\mathcal{A}_{p}(E)$. Since any topological vector bundle admits a compatible smooth structure, we may regard $L_{p}^{2}$ connections as those that differ from a smooth connection by a $L_{p}^{2} 1$-form. In other words, $\mathcal{A}_{p}(E)$ becomes an affine space modeled over the Hilbert space of $L_{p}^{2} 1$-forms with values in the endomorphisms of $E$. The curvature of a connection in $\mathcal{A}_{p}(E)$ then becomes a $L_{p-1}^{2} 2$-form with values in the endomorphism bundle $\operatorname{End}(E)$. Moreover, let $\mathcal{G}_{p+1}(E)$ be defined as the topological group of all $L_{p+1}^{2}$ bundle automorphisms. By virtue of the Sobolev multiplication theorem, $\mathcal{G}_{p+1}(E)$ has the structure of an infinite dimensional Lie group modeled on a Hilbert space; its Lie algebra is the space of $L_{p+1}^{2}$ sections of $\operatorname{End}(E)$. 
The Sobolev multiplication theorem is once again invoked to guarantee that the action $\mathcal{G}_{p+1}(E) \times \mathcal{A}_{p}(E) \rightarrow \mathcal{A}_{p}(E)$ is a smooth map of Hilbert manifolds. The quotient space $\mathcal{B}_{p}(E)=\mathcal{A}_{p}(E) / \mathcal{G}_{p+1}(E)$ inherits a topological structure; it is a metric (hence Hausdorff) topological space. Therefore, the subspace $\mathcal{M}_{X}(E)$ of $\mathcal{B}_{p}(E)$ is also a Hausdorff topological space; moreover, one can show that the topology of $\mathcal{M}_{X}(E)$ does not depend on $p$.

The quotient space $\mathcal{B}_{p}(E)$ fails to be a Hilbert manifold because in general the action of $\mathcal{G}_{p+1}(E)$ on $\mathcal{A}_{p}(E)$ is not free. Indeed, if $A$ is a connection on a rank $r$ complex vector bundle $E$ over a connected base manifold $X$, which is associated with a principal $G$-bundle. Then the isotropy group of $A$ within the gauge group:

$$
\Gamma_{A}=\left\{g \in \mathcal{G}_{p+1}(E) \mid g(A)=A\right\}
$$

is isomorphic to the centralizer of the holonomy group of $A$ within $G$.

This means that the subspace of irreducible connections $\mathcal{A}_{p}^{*}(E)$ can be equivalently defined as the open dense subset of $\mathcal{A}_{p}(E)$ consisting of those connections whose isotropy group is minimal, that is:

$$
\mathcal{A}_{p}^{*}(E)=\left\{A \in \mathcal{A}_{p}(E) \mid \Gamma_{A}=\operatorname{center}(G)\right\} .
$$

Now $\mathcal{G}_{p+1}(E)$ acts with constant isotropy on $\mathcal{A}_{p}^{*}(E)$, hence the quotient $\mathcal{B}_{p}^{*}(E)=$ $\mathcal{A}_{p}^{*}(E) / \mathcal{G}_{p+1}(E)$ acquires the structure of a smooth Hilbert manifold.

Remark. The analysis of neighborhoods of points in $\mathcal{B}_{p}(E) \backslash \mathcal{B}_{p}^{*}(E)$ is very relevant for applications of the instanton moduli spaces to differential topology. The simplest situation occurs when $A$ is an $S U(2)$-connection on a rank 2 complex vector bundle $E$ which reduces to a pair of $U(1)$ and such $[A]$ occurs as an isolated point in $\mathcal{B}_{p}(E) \backslash \mathcal{B}_{p}^{*}(E)$. Then a neighborhood of $[A]$ in $\mathcal{B}_{p}(E)$ looks like a cone on an infinite dimensional complex projective space.

Alternatively, the instanton moduli space $\mathcal{M}_{X}(E)$ can also be described by first taking the subset of all anti-self-dual connections and then taking the quotient under the action of the gauge group. More precisely, consider the map:

$$
\begin{gathered}
\rho: \mathcal{A}_{p}(E) \rightarrow L_{p}^{2}\left(\operatorname{End}(E) \otimes \Omega_{X}^{2,+}\right) \\
\rho(A)=F_{A}^{+}
\end{gathered}
$$

Thus $\rho^{-1}(0)$ is exactly the set of all anti-self-dual connections. It is $\mathcal{G}_{p+1}(E)$ invariant, so we can take the quotient to get:

$$
\mathcal{M}_{X}(E)=\rho^{-1}(0) / \mathcal{G}_{p+1}(E) .
$$

It follows that the subspace $\mathcal{M}_{X}^{*}(E)=\mathcal{B}_{p}^{*}(E) \cap \mathcal{M}_{X}(E)$ has the structure of a smooth Hilbert manifold. Index theory comes into play to show that $\mathcal{M}_{X}^{*}(E)$ is finite-dimensional. Recall that if $D$ is an elliptic operator on a vector bundle over a compact manifold, then $D$ is Fredholm (i.e. $\operatorname{ker} D$ and coker $D$ are finite dimensional) and its index

$$
\text { ind } D=\operatorname{dim} \operatorname{ker} D-\operatorname{dim} \operatorname{coker} D
$$


can be computed in terms of topological invariants, as prescribed by the AtiyahSinger index theorem. The goal here is to identify the tangent space of $\mathcal{M}_{X}^{*}(E)$ with the kernel of an elliptic operator.

It is clear that for each $A \in \mathcal{A}_{p}(E)$, the tangent space $T_{A} \mathcal{A}_{p}(E)$ is just $L_{p}^{2}(\operatorname{End}(E) \otimes$ $\left.\Omega_{X}^{1}\right)$. We define the pairing:

$$
\langle a, b\rangle=\int_{X} a \wedge * b
$$

and it is easy to see that this pairing defines a Riemannian metric (so-called $L^{2}$-metric) on $\mathcal{A}_{p}(E)$.

The derivative of the map $\rho$ in (17) at the point $A$ is given by:

$$
\begin{aligned}
d_{A}^{+}: L_{p}^{2}\left(\operatorname{End}(E) \otimes \Omega_{X}^{1}\right) \rightarrow L_{p-1}^{2}\left(\operatorname{End}(E) \otimes \Omega_{X}^{2}\right) \\
a \mapsto\left(d_{A} a\right)^{+},
\end{aligned}
$$

so that for each $A \in \rho^{-1}(0)$ we have:

$$
T_{A} \rho^{-1}(0)=\left\{a \in L_{p}^{2}(\operatorname{End}(E)) \otimes \Omega_{X}^{1} \mid d_{A}^{+} a=0\right\} .
$$

Now for a gauge equivalence class $[A] \in \mathcal{B}_{p}^{*}(E)$, the tangent space $T_{[A]} \mathcal{B}_{p}^{*}(E)$ consists of those 1-forms which are orthogonal to the fibers of the principal $\mathcal{G}_{p+1}(E)$ bundle $\mathcal{A}_{p}^{*}(E) \rightarrow \mathcal{B}_{p}^{*}(E)$. At a point $A \in \mathcal{A}_{p}(E)$, the derivative of the action by some $g \in \mathcal{G}_{p+1}(E)$ is

$$
-d_{A}: L_{p+1}^{2}(\operatorname{End}(E)) \rightarrow L_{p}^{2}\left(\operatorname{End}(E) \otimes \Omega_{X}^{1}\right) .
$$

Usual Hodge decomposition gives us that there is an orthogonal decomposition:

$$
L_{p}^{2}\left(\operatorname{End}(E) \otimes \Omega_{X}^{1}\right)=\operatorname{im} d_{A} \oplus \operatorname{ker} d_{A}^{*}
$$

which means that:

$$
T_{[A]} \mathcal{B}_{p}^{*}(E)=\left\{a \in L_{p}^{2}\left(\operatorname{End}(E) \otimes \Omega_{X}^{1}\right) \mid d_{A}^{*} a=0\right\} .
$$

Thus the pairing (18) also defines a Riemannian metric on $\mathcal{B}_{p}^{*}(E)$.

Putting these together, we conclude that the space $T_{[A]} \mathcal{M}_{X}^{*}(E)$ tangent to $\mathcal{M}_{X}^{*}(E)$ at an equivalence class $[A]$ of anti-self-dual connections can be described as follows:

$$
T_{[A]} \mathcal{M}_{X}^{*}(E)=\left\{a \in L_{p}^{2}\left(\operatorname{End}(E) \otimes \Omega_{X}^{1}\right) \mid d_{A}^{*} a=d_{A}^{+} a=0\right\}
$$

It turns out that the so-called deformation operator $\delta_{A}=d_{A}^{*} \oplus d_{A}$ :

$$
\delta_{A}: L_{p}^{2}\left(\operatorname{End}(E) \otimes \Omega_{X}^{1}\right) \rightarrow L_{p+1}^{2}(\operatorname{End}(E)) \oplus L_{p-1}^{2}\left(\operatorname{End}(E) \otimes \Omega_{X}^{2}\right)
$$

is elliptic. Moreover, if $A$ is anti-self-dual then coker $\delta_{A}$ is empty, so that $T_{[A]} \mathcal{M}_{X}^{*}(E)=\operatorname{ker} \delta_{A}$. The dimension of the tangent space $T_{[A]} \mathcal{M}_{X}^{*}(E)$ is then 
simply given by the index of the deformation operator $\delta_{A}$. Using the AtiyahSinger index theorem, we have for $S U(r)$-bundles with $c_{2}(E)=k$ :

$$
\operatorname{dim} \mathcal{M}_{X}^{*}(E)=4 r k-\left(r^{2}-1\right)\left(1-b_{1}(X)+b_{+}(X)\right) .
$$

The dimension formula for arbitrary gauge group $G$ can be found at [1].

For example, the moduli space of $S U(2)$ instantons on $\mathbb{R}^{4}$ of charge $k$ is a smooth Riemannian manifold of dimension $8 k-3$. These parameters are interpreted as the $5 k$ parameters describing the positions and sizes of $k$ separate instantons, plus $3(k-1)$ parameters describing their relative $S U(2)$ phases.

The detailed construction of the instanton moduli spaces can be found at [4] An alternative source is Morgan's lecture notes in [5].

It is interesting to note that $\mathcal{M}_{X}^{*}(E)$ inherits many of the geometrical properties of the original manifold $X$. Most notably, if $X$ is a Kähler manifold, then $\mathcal{M}_{X}^{*}(E)$ is also Kähler; if $X$ is a hyperkähler manifold, then $\mathcal{M}_{X}^{*}(E)$ is also hyperkähler. One expects that other geometric structures on $X$ can also be transfered to the instanton moduli spaces $\mathcal{M}_{X}^{*}(E)$.

\section{References}

[1] M. F. Atiyah, N. J. Hitchin \& I. M. Singer. Self-duality in four-dimensional riemannian geometry. Proc. Royal Soc. London 362, 425-461 (1978).

[2] C. W. Bernard, N. H. Christ, A. H. Guth \& E. J. Weinberg. Pseudoparticle parameters for arbitrary gauge groups. Phys. Rev. D 16, 2967-2977 (1977).

[3] J. P. Bourguignon \& H. B. Lawson Jr. Stability and isolation phenomena for Yang-Mills fields Commun. Math. Phys. 79 189-230 (1981).

[4] S. K. Donaldson \& P. B. Kronheimer. Geometry of four-manifolds. Oxford: Clarendon Press 1990.

[5] R. Friedman \& J. W. Morgan (eds.) Gauge theory and the topology of four-manifolds. Providence, RI: American Mathematical Society 1998.

[6] N. Hitchin. The self-duality equations on a Riemann surface. Proc. London Math. Soc. 55, 59-126 (1987).

Stable bundles and integrable systems. Duke Math. J. 54, 91-114 (1987).

[7] M. Jardim. A survey on Nahm transform. J. Geom. Phys. 52, 313-327 (2004).

[8] L. J. Mason \& N. M. J. Woodhouse. Integrability, self-duality, and twistor theory. New York, NY: Clarendon Press 1996.

[9] M. Murray. Monopoles. In: Geometric analysis and applications to quantum field theory, 119-135. Progr. Math. 205. Boston, MA: Birkhuser Boston 2002. 